\title{
A NEW METHOD OF INTEGRATION BY MEANS OF ORTHOGONALITY FOCI*
}

\author{
BY
}

\author{
A. A. POPOFF
}

Automechanical Institute, Moscow, U.S.S.R.

1. Introduction. This paper contains a new method of integration which is partly graphical, partly analytical. ${ }^{1}$ It permits a simple determination of integrals of the form $\int \phi_{i}(x) \phi_{k}(x) d x$, where $\phi_{i}(x)$ is given graphically and $\phi_{k}(x)$ is given either graphically or analytically. The method requires the construction of certain diagrams, called scales, showing the abscissae of the centroids of certain areas associated with $\phi_{k}(x)$, and is based on some properties of the so-called orthogonality foci. Finally, the method is applied to interpolation, Fourier analysis, and the evaluation of Mohr integrals in the theory of structures.

2. Definite integrals. Let us consider the integral

$$
T=\int_{0}^{l} \phi_{i}(x) \phi_{k}(x) d x .
$$

If rectangular cartesian coordinates $x, y$ are introduced, the functions $\phi_{i}(x)$, $\phi_{k}(x)$ can be represented by curves, such as in Fig. 1. We now consider a distribution of mass along the curve $y=\phi_{i}(x)$, $0 \leqq x \leqq l$, the mass per unit length in the $x$-direction being $\phi_{k}(x)$. The centroid of this mass distribution we shall call the orthogonality focus. ${ }^{2}$ We shall denote it by $F_{i k}$, and its coordinates by $\rho_{k}, f_{i k}$ (neither $\rho_{k}$ nor the total mass $\Omega_{k}$ of the system depend on $\left.\phi_{i}(x)\right)$. We have

$$
\Omega_{k}=\int_{0}^{l} \phi_{k}(x) d x
$$

$\Omega_{k}$ is also the area under the curve $y=\phi_{k}(x)$. Since $T$ represents the mass moment of the mass distribution about the $x$-axis,

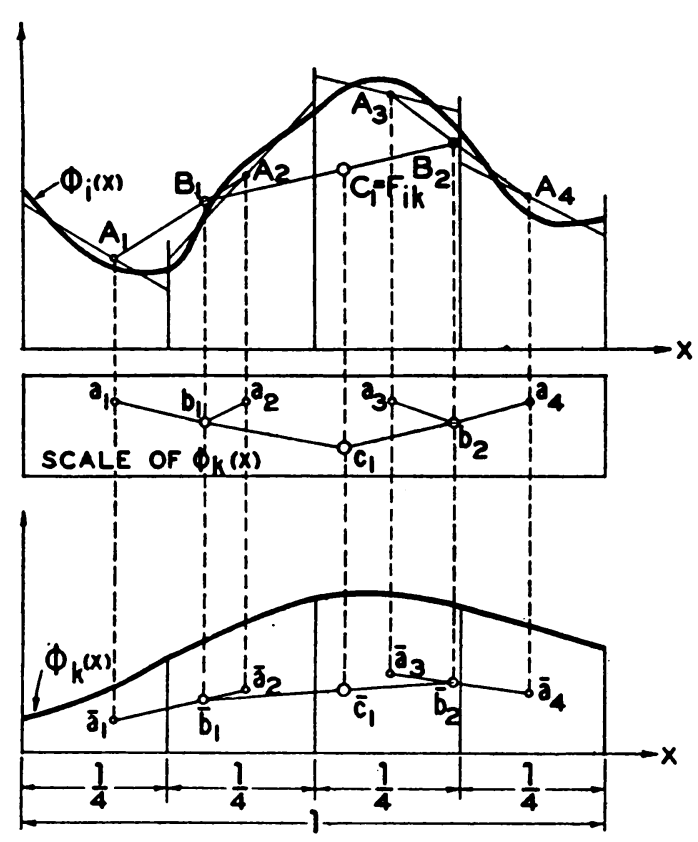

Fig. 1.

* Russian manuscript received Feb. 24, 1944. The present condensed version was prepared by Dr. L. Bers, Brown University, and Professor G. E. Hay, University of Michigan.

${ }^{1}$ This method was announced in the author's note entitled $A$ new method of graphical integration, C. R. (Doklady) Acad. Sci. URSS (N.S.) 38, (1943).

2 This name is justified by the properties discussed in Section 4. 


$$
\rho_{k}=\frac{1}{\Omega_{k}} \int_{0}^{l} x \phi_{k}(x) d x,
$$

$$
f_{i k}=T / \Omega_{k}
$$

$\rho_{k}$ is also the abscissa of the centroid of the area under the curve $y=\phi_{k}(x)$.

The following lemmas can be verified easily:

(a) If $\phi_{i}(x)$ is a linear function, its graph is a straight line and $F_{i k}$ lies on this line; $F_{i k}$ can thus be found immediately if $\rho_{k}$ is known.

(b) If the interval $(0, l)$ is divided into two parts, the orthogonal foci of the two parts and of the whole are collinear.

These two lemmas permit a graphical determination to any desired degree of accuracy of the point $F_{i k}$ and hence of the integral $T$. The procedure is as follows:

(a) The in terval $(0, l)$ is divided in to $2^{m}$ equal in tervals ${ }^{3}\left(0, l_{1}\right),\left(l_{1}, l_{2}\right), \cdots,\left(l_{2}{ }_{-1}, l\right)$.

(b) Operation (a) divides the region under the curve $y=\phi_{k}(x)$ into $2^{m}$ regions. We find the centroids $\bar{a}_{r}(r=1$, $\left.2, \cdots, 2^{m}\right)$ of these $2^{m}$ regions, then combine adjacent pairs of regions and find the centroids $b_{r}$ $\left(r=1,2, \cdots, 2^{m-1}\right)$ of the $2^{m-1}$ regions so formed, then combine adjacent pairs of these $2^{m-1}$ regions and find the centroids $\bar{c}_{r}$ $\left(r=1,2, \cdots, 2^{m-2}\right)$ of the $2^{m-2}$ regions so formed, and so on. In the final stage, we find the centroid of the entire region under the curve $y=\phi_{k}(x)$. In the lower part of Fig. 1, we see these centroids in a case when $m=2$.

(c) A diagram, called the scale of $\phi_{k}(x)$, is constructed. The mid-

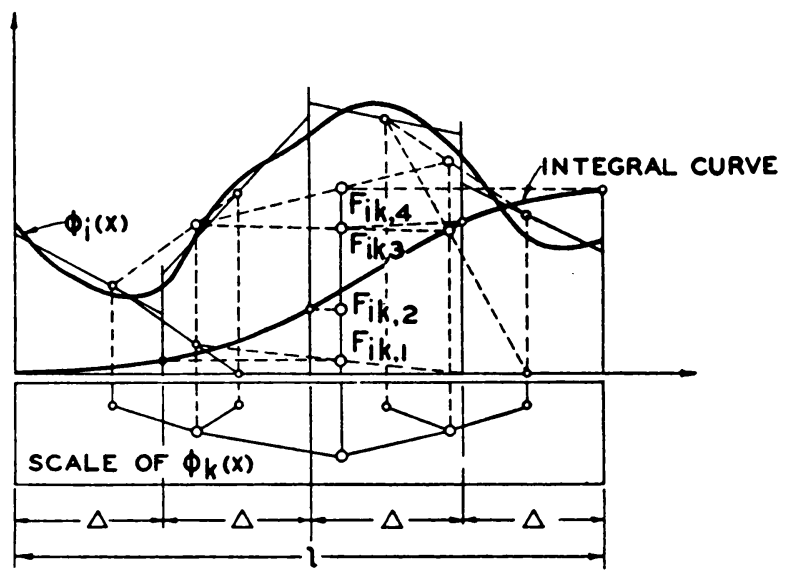

FIG. 2. dle part of Fig. 1 shows such a scale. It consists of points $a_{r}\left(r=1,2, \cdots, 2^{m}\right)$ vertically above $\bar{a}_{r}$ and all at the same level, points $b_{r}\left(r=1,2, \cdots, 2^{m-1}\right)$ vertically above $b_{r}$ and all at the same arbitrary level slightly below the points $a_{r}$, and so on.

(d) Operation (a) divides the curve $y=\phi_{i}(x)$ into $2^{m}$ parts. We replace each part by a segment of a straight line, as shown in the upper part of Fig. 1, and assume that the mass is distributed along these segments rather than along the curve.

(e) We determine the points $A_{r}\left(r=1,2, \cdots, 2^{m}\right)$ of intersection of these line segments with the verticals through the points $a_{r}$. We then determine the points $B_{r}\left(r=1,2, \cdots, 2^{m-1}\right)$ of intersection of the straight lines joining adjacent pairs of points $A_{r}$ with verticals through the points $b_{r}$. This process is repeated, until finally we arrive at the final point $F_{i k}$.

(f) $f_{i k}$, which is the ordinate of $F_{i k}$, is determined by measurement; the value $T$ of the required integral then follows from (2.4).

\footnotetext{
3 Unequal intervals could also be used.

4 It is to be noted that areas corresponding to negative values of $\phi_{k}(x)$ must be considered as corresponding to negative mass.
} 
3. Indefinite integrals. The graphical construction of Section 2 can be applied to the indefinite integral $\int \phi_{i}(x) \phi_{k}(x) d x$, in the following manner (see Fig. 2). An interval $(0, l)$ on the $x$-axis is taken, and the construction of Section 2 is applied to the integral $\int_{0}^{l} \phi_{i, 1}(x) \phi_{k}(x) d x$, where $\phi_{i, 1}(x)=\phi_{i}(x)$ in $\left(0, l_{1}\right)$ and vanishes elsewhere. This yields a point $F_{i k, 1}$ with ordinate $f_{i k, 1}$. The point $\left(l_{1}, f_{i k, 1}\right)$ is then plotted. The construction of Section 2 is then applied to the integral $\int_{0}^{l} \phi_{i, 2}(x) \phi_{k}(x) d x$, where $\phi_{i, 2}(x)=\phi_{i}(x)$ in $\left(0, l_{2}\right)$ and vanishes elsewhere. This yields a point $F_{i k, 2}$, and the point $\left(l_{2}, f_{i k, 2}\right)$ is plotted. In this way we obtain the sequence of points $\left(l_{r}, f_{i k, r}\right)$, $\left(r=1,2, \cdots, 2^{m}\right)$. Since

$$
f_{i k, r}=\frac{1}{\Omega_{k}} \int_{0}^{l} \phi_{i, r}(x) \phi_{k}(x) d x,
$$

the curve passing through these points is approximately the integral curve, except for the constant factor $1 / \Omega_{k}$.

Figure 2 shows this construction for the same functions $\phi_{i}(x), \phi_{k}(x)$ considered in Fig. $1, m$ again having the value 2 .

4. Some properties of orthogonality foci. (a) If the $x$-axis passes through the point $F_{i k}$, then $f_{i k}=0$, and by $(2.4)$

$$
\int_{0}^{l} \phi_{i}(x) \phi_{k}(x) d x=0,
$$

i.e., $\phi_{i}(x)$ and $\phi_{k}(x)$ are orthogonal. It is for this reason that $F_{i k}$ is called the orthogonality focus.

(b) Let us set $\phi_{i}(x) \equiv \phi_{k}(x)$. Then, from (2.4),

$$
f_{k k}=\frac{1}{\Omega_{k}} \int_{0}^{l}\left[\phi_{k}(x)\right]^{2} d x=2 f_{k}
$$

where $f_{k}$ is the ordinate of the centroid of the region under the curve $y=\phi_{k}(x)$, $(0 \leqq x \leqq l)$. We shall now prove the following theorem. The curve $y=h \phi_{k}(x)$, where $h$ is a constant, has the least mean square deviation from the curve $y=\phi_{i}(x)$ when

$$
h=f_{i k} / f_{k k} .
$$

To prove this, we note that the mean square deviation is a minimum when

$$
\frac{d}{d h} \int_{0}^{l}\left[\phi_{i}(x)-h \phi_{k}(x)\right]^{2} d x=0,
$$

i.e., when

$$
\int_{0}^{l}\left[\phi_{i}(x)-h \phi_{k}(x)\right] \phi_{k}(x) d x=0,
$$

or

$$
h=\int_{0}^{l} \phi_{i}(x) \phi_{k}(x) d x / \int_{0}^{l}\left[\phi_{k}(x)\right]^{2} d x=f_{i k} / f_{k k} .
$$


(c) Let us draw the horizontal line $\beta$ through the point $F_{i k}$ (Fig. 3), and then rotate $\beta$ about $F_{i k}$ through an angle $\alpha$ to a new position $\beta^{\prime}$. A new curve $y=\phi_{i}^{\prime}(x)$ is constructed such that the vertical distance from $\beta^{\prime}$ to points on this curve is equal to the vertical distance from $\beta$ to points on the curve $y=\phi_{i}(x)$. We shall now prove that

$$
\int_{0}^{l} \phi_{i}^{\prime}(x) \phi_{k}(x) d x=\int_{0}^{l} \phi_{i}(x) \phi_{k}(x) d x .
$$

We have $\phi_{i}^{\prime}(x)=\phi_{i}(x)+\left(\rho_{k}-x\right) \tan \alpha$, where, it is recalled, $\rho_{k}$ is the abscissa of $F_{i k}$. Thus

$$
\int_{0}^{l} \phi_{i}^{\prime}(x) \phi_{k}(x) d x=\int_{0}^{l} \phi_{i}(x) \phi_{k}(x) d x+\tan \alpha \int_{0}^{l}\left(\rho_{k}-x\right) \phi_{k}(x) d x .
$$

The last integral vanishes, by the definition of $\rho_{k}$, and the desired result is obtained.

It is to be noted that the two curves have a common point $I$, about which the curve is "rotated."

(d) Let us consider the case when $\phi_{i}(x)$ is linear in each of the intervals $\left(0, l_{1}\right)\left(l_{1}, l\right)$, so that its graph is a broken line $a b c d$ (Fig. 4). We shall now show that, if $a b$ is rotated about $a$ point $M$ on $a b$ to a new position $a^{\prime} b^{\prime}$, then $F_{i k}$ is unchanged if $c d$ is rotated about a certain point $N$ on cd in such a way that $b c=b^{\prime} c^{\prime}$. The points $M, N$ are called conjugate foci. To prove this theorem, we use Fig. 4, in which $A_{1}, A_{2}, A_{1}^{\prime}, A_{2}^{\prime}$ are points leading to the determination of $F_{i k}$, following the procedure laid down in operation

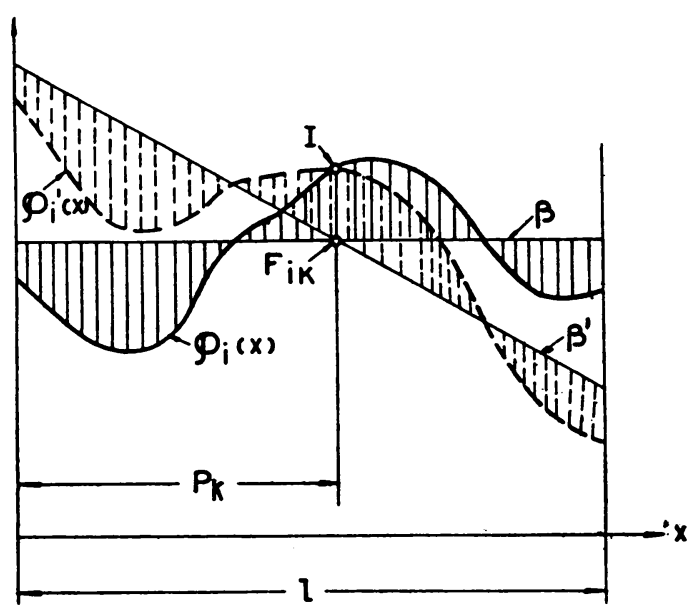

FIG. 3. (e) of Section 2. From the three pairs of similar triangles $M b b^{\prime}$ and $M A_{1} A_{1}^{\prime}, N c c^{\prime}$ and $N A_{2} A_{2}^{\prime}, F_{i k} A_{1} A_{1}^{\prime}$ and $F_{i k} A_{2} A_{2}^{\prime}$, we have

$$
\frac{b b^{\prime}}{A_{1} A_{1}^{\prime}}=\frac{\beta_{1}}{\beta_{1}-\gamma_{1}}, \quad \frac{c c^{\prime}}{A_{2} A_{2}^{\prime}}=\frac{\beta_{2}}{\gamma_{2}-\beta_{2}}, \quad \frac{A_{1} A_{1}^{\prime}}{A_{2} A_{2}^{\prime}}=\frac{\alpha_{1}}{\alpha_{2}} .
$$

Since $b b^{\prime}=c c^{\prime}$, a value for $A_{1} A_{1}^{\prime} / A_{2} A_{2}^{\prime}$ can be determined from the first two equations. Substitution of this value in the third equation yields

$$
\alpha_{1} \gamma_{2}\left(1 / \beta_{2}\right)+\alpha_{2} \gamma_{1}\left(1 / \beta_{1}\right)=\alpha_{1}+\alpha_{2} .
$$

Thus $\beta_{2}$ is uniquely determined by $\beta_{1}$; hence $N$ is uniquely determined by $M$.

It is easily seen that, if rotations of the above type are carried out about conjugate foci, and if $\phi_{i}^{\prime}(x)$ denotes the function the graph of which is $a^{\prime} b^{\prime} c^{\prime} d^{\prime}$, then

$$
\int_{0}^{l} \phi_{i}^{\prime}(x) \phi_{k}(x) d x=\int_{0}^{l} \phi_{i}(x) \phi_{k}(x) d x .
$$




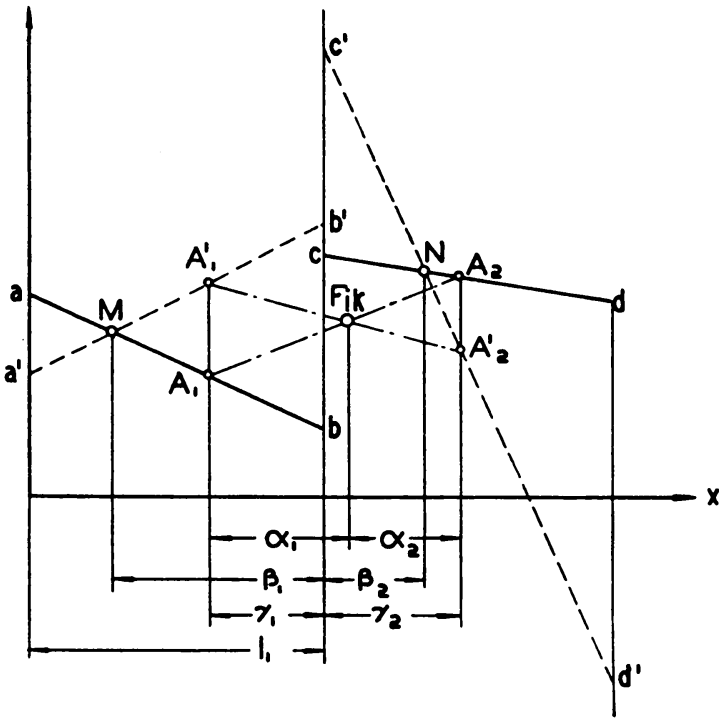

FIG. 4.

(e) Let us denote by $F_{i k}$ and $F_{i l}$ the orthogonal foci of the function $\phi_{i}(x)$ represented by the broken line $a b c d$ with respect to two functions $\phi_{k}(x)$ and $\phi_{l}(x)$. We shall now show that, there is a unique pair of conjugate foci $M$ (on $a b$ ) and $N$ (on $c d$ ) such that rotations of $a b$ about $M$ and $c d$ about $N$ (with $b c=b^{\prime} c^{\prime}$ ) leave both $F_{i k}$ and $F_{i l}$ unchanged. This follows from the fact that $F_{i k}$ is unchanged if $\beta_{1}$ and $\beta_{2}$ (Fig. 4) satisfy (4.5), and $F_{i l}$ is unchanged if they satisfy a second relation of the same form as (4.5). Since both these relations are linear in $1 / \beta_{1}, 1 / \beta_{2}$, they can be solved for unique values of $\beta_{1}$ and $\beta_{2}$.

It is easily seen that, if rotations of the above type are carried out about such conjugate foci, and if $\phi_{i}^{\prime}(x)$ denotes the function the graph of which is displaced position of $a b c d$, then (4.6) holds and also

$$
\int_{0}^{l} \phi_{i}^{\prime}(x) \phi_{l}(x) d x=\int_{0}^{l} \phi_{i}(x) \phi_{l}(x) d x .
$$

Conjugate foci can be used widely in graphical computations dealing with statically indeterminate structures.

5. Application of orthogonality foci to the interpolation of curves. Let us consider the application of orthogonality foci to the following problem. We are given two functions or curves $\phi_{i}(x)$ and $\phi_{k}(x)$. It is required to find a straight line $y=A+B x$ such that the integral

$$
U=\int_{0}^{l}\left[\phi_{i}-(A+B x)\right]^{2} \phi_{k} d x,
$$

will have the least possible value. We shall now show that $U$ has the least possible value when the straight line $y=A+B x$ passes through the orthogonality foci $F_{i k}, F_{i l}$, where $\phi_{l}=x \phi_{k}$.

We set $\partial U / \partial A=\partial U / \partial B=0$, to obtain the equations

$$
\left.\begin{array}{l}
A \int_{0}^{l} \phi_{k} d x+B \int_{0}^{l} x \phi_{k} d x=\int_{0}^{l} \phi_{i} \phi_{k} d x, \\
A \int_{0}^{l} x \phi_{k} d x+B \int_{0}^{l} x^{2} \phi_{k} d x=\int_{0}^{l} x \phi_{i} \phi_{k} d x .
\end{array}\right\}
$$

Let us consider the functions $\phi_{0}(x)=1, \phi_{1}(x)=x, \phi_{2}(x)=x^{2}$. We have $\Omega_{0}=l, \Omega_{1}=\frac{1}{2} l^{2}$, $\Omega_{2}=\frac{1}{3} l^{3}$. If the orthogonality foci of $\phi_{k}$ with $\phi_{0}, \phi_{1}, \phi_{2}$ are denoted by $F_{k 0}\left(\rho_{0}, f_{k 0}\right)$, $F_{k 1}\left(\rho_{1}, f_{k 1}\right), F_{k 2}\left(\rho_{2}, f_{k 2}\right)$, respectively, and the orthogonality foci of $\phi_{i}$ with $\phi_{k}$ and $\phi_{l}$ are denoted by $F_{i k}\left(\rho_{k}, f_{i k}\right), F_{i l}\left(\rho_{l}, F_{i l}\right)$, respectively, then 


$$
\begin{gathered}
\int_{0}^{l} \phi_{k} d x=f_{k 0} l, \quad \int_{0}^{l} \phi_{k} x d x=\frac{1}{2} f_{k 1} l^{2}, \quad \int_{0}^{l} \phi_{k} x^{2} d x=\frac{1}{3} f_{k 2} l^{3} \\
\int_{0}^{l} \phi_{i} \phi_{k} d x=f_{i k} \int_{0}^{l} \phi_{k} d x=f_{i k} f_{k 0} l \\
\int_{0}^{l} \phi_{i} \phi_{l} d x=f_{i l} \int_{0}^{l} \phi_{k} x d x=\frac{1}{2} f_{i l} f_{k \cdot 1} l^{2}
\end{gathered}
$$

Thus (5.2) can be written in the form

$$
A+B \frac{f_{k 1} l}{2 f_{k 0}}=f_{i k}, \quad A+B \frac{2 f_{k 2} l}{3 f_{k 1}}=f_{i l} .
$$

Since $\rho_{k}, \rho_{l}$ are abscissas of orthogonality foci, by (2.3) we have

$$
\left.\begin{array}{rl}
\rho_{k} & =\int_{0}^{l} x \phi_{k} d x / \int_{0}^{l} \phi_{k} d x=\frac{f_{k 1} l}{f_{k 0}}, \\
\rho_{l} & =\int_{0}^{l} x^{2} \phi_{k} d x / \int_{0}^{l} x \phi_{k} d x=\frac{2 f_{k 2} l}{3 f_{k 1}} .
\end{array}\right\}
$$

Thus (5.5) take the form

$$
A+B \rho_{k}=f_{i k}, \quad A+B \rho_{l}=f_{i l},
$$

whence it follows that $A$ and $B$ must be such that the straight line $y=A+B x$ passes through the orthogonality foci $F_{i k}, F_{i l}$.

In order to construct the straight line $y=A+B x$ which is such that $U$ has the least possible value, we can proceed as follows:

(a) Scales are constructed for $\phi_{0}=1, \phi_{1}=x, \phi_{2}=x^{2}$. These are as shown in Fig. 5 when the interval $(0, l)$ is divided into 8 equal parts $(m=3)$.

(b) A scale is constructed for $\phi_{k}$. If the function $\phi_{k}$ is given, this can be done analytically. In any event, it can be done graphically using the scales in Fig. 5 , since it involves integrals of the forms $\int \phi_{k} d x, \int \phi_{k} x d x$.

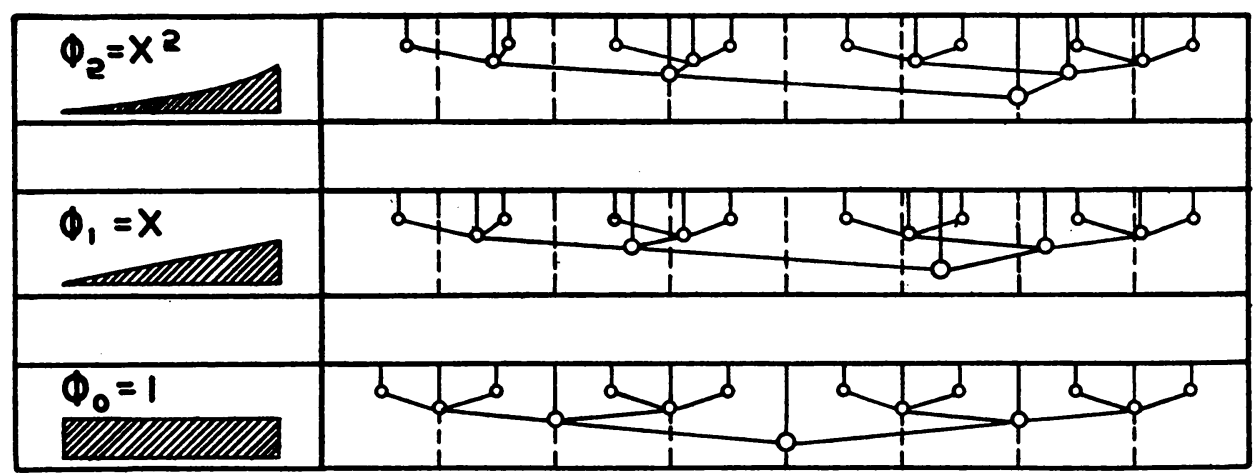

FIG. 5. 


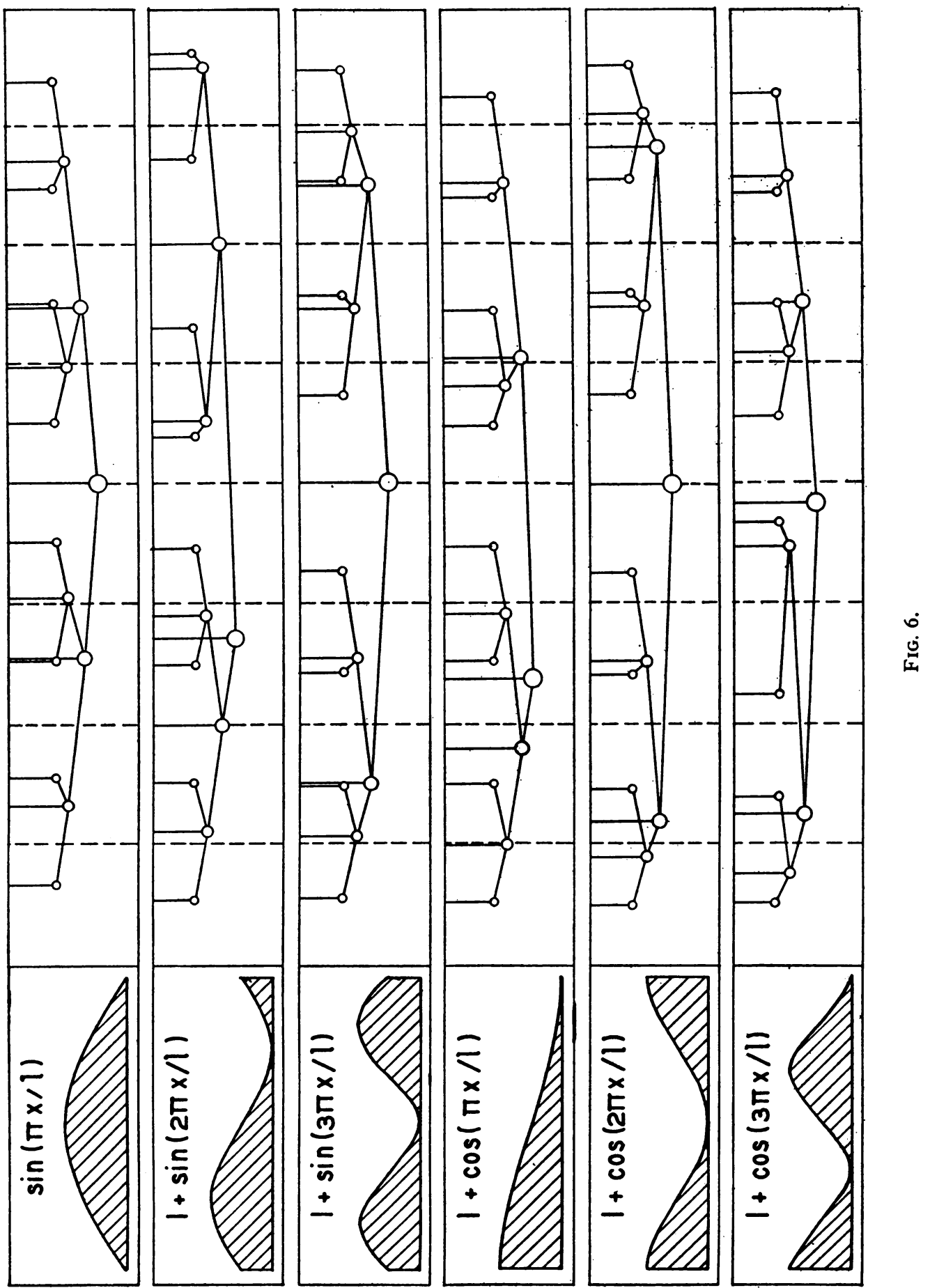


(c) A scale is constructed for $\phi_{l}=x \phi_{k}$. This also can be done graphically by means of the scales in Fig. 5.

(d) The foci $F_{i k}, F_{i l}$ are found following the procedure outlined in Section 2. The straight line through $F_{i k}, F_{i l}$ is the required line.

6. Graphical harmonic analysis. Orthogonality foci can be used to obtain the Fourier series expansion of a function which is given either analytically or graphically. If $\phi(x)$ denotes the function, its expansion in to a Fourier series of sines or cosines will involve the integrals

$$
\left.\begin{array}{rl}
a_{n}=\frac{2}{l} \int_{0}^{l} \phi(x) \sin \frac{n \pi x}{l} d x & (n=1,2, \cdots), \\
b_{n}=\frac{2}{l} \int_{0}^{l} \phi(x) \cos \frac{n \pi x}{l} d x & (n=0,1, \cdots),
\end{array}\right\}
$$

where $a_{n}, b_{n}$ are Fourier coefficients.

Difficulties are encountered if the method of orthogonal foci is applied directly to the integrals in (6.1). These difficulties are avoided if we write (6.1) in the form

where

$$
\left.\begin{array}{ll}
a_{1}=\frac{2}{l} \int_{0}^{l} \phi(x) \phi_{s 1}(x) d x, & \\
a_{n}=\frac{2}{l} \int_{0}^{l} \phi(x) \phi_{s n}(x) d x-\frac{2}{l} \int_{0}^{l} \phi(x) d x & (n=2,3, \cdots), \\
b_{n}=\frac{2}{l} \int_{0}^{l} \phi(x) \phi_{c n}(x) d x-\frac{2}{l} \int_{0}^{l} \phi(x) d x & (n=0,1, \cdots),
\end{array}\right\}
$$

$$
\begin{aligned}
& \phi_{s 1}(x)=\sin \frac{\pi x}{l}, \quad \phi_{s n}(x)=1+\sin \frac{n \pi x}{l} \quad(n=2,3, \cdots), \\
& \phi_{c n}(x)=\cos \frac{n \pi x}{l} \quad(n=0,1, \cdots) .
\end{aligned}
$$

By the use of the scale of $\phi_{0}(z)=1$ (Fig. 5) and the scales of the functions in (6.3) (Fig. 6), the ordinates of the orthogonality foci of $\phi(x)$ with these functions can be found graphically by the procedure of Section 2. If we denote these ordinates by $f_{0}, f_{s n}, f_{c n}$, respectively, then (6.2) takes the form

$$
\begin{aligned}
& a_{1}=\frac{2}{l} f_{s 1} \Omega_{s 1}, \quad a_{n}=\frac{2}{l}\left(f_{s n} \Omega_{s n}-f_{0} \Omega_{0}\right) \quad(n=2,3, \cdots), \\
& b_{n}=\frac{2}{l}\left(f_{c n} \Omega_{c n}-f_{0} \Omega_{0}\right) \quad(n=0,1, \cdots),
\end{aligned}
$$

where $\Omega_{0}, \Omega_{s n}, \Omega_{c n}$ are respectively the areas under the curve $\phi_{0}(x)=1$ and the curves in $(6.3)$ for the interval $(0, l)$. Now

$$
\begin{aligned}
& \Omega_{s 1}=\frac{2 l}{\pi}, \quad \Omega_{s n}=l\left(1+\frac{1-\cos n \pi}{n \pi}\right) \quad(n=2,3, \cdots), \\
& \Omega_{0}=l, \quad \Omega_{c n}=l,
\end{aligned}
$$


whence (6.4) becomes

$$
\begin{aligned}
& a_{1}=\frac{4}{\pi} f_{s 1}, \quad a_{n}=2\left[f_{s n}\left(1+\frac{1-\cos n \pi}{n \pi}\right)-f_{0}\right] \quad(n=2,3, \cdots), \\
& b_{n}=2\left(f_{c n}-f_{0}\right) \quad(n=0,1, \cdots) .
\end{aligned}
$$

7. Graphical evaluation of Mohr integrals. In the theory of structures, the determination of deflections in bending of ten requires the evaluation of so-called Mohr

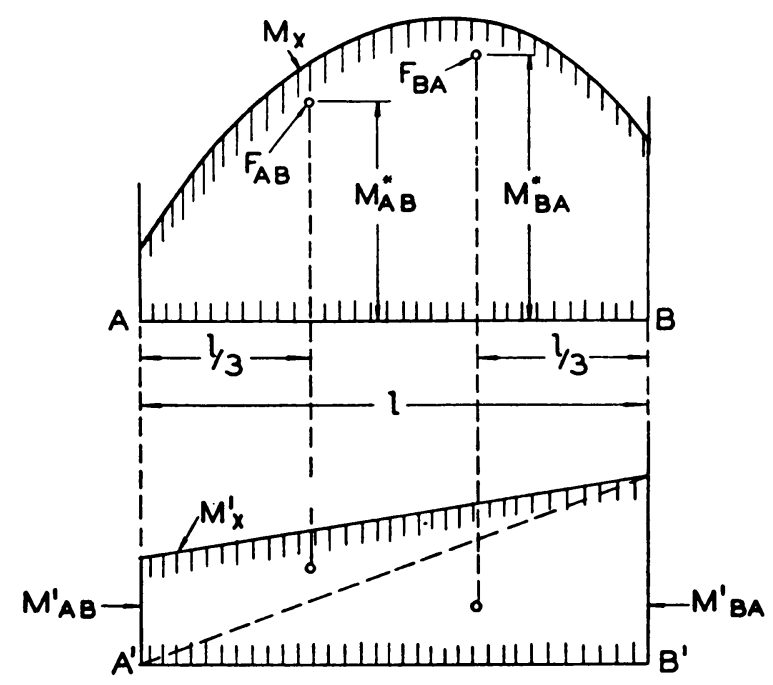

FIG. 7.

integrals, which have the form $T=\int_{0}^{l} M_{x} M_{x}^{\prime} d x$, where $M_{x}$ is a function of $x$.given graphically and $M_{x}^{\prime}$ is a linear function of $x$ (Fig. 7).

From Fig. 7, we see that

Thus

$$
M_{x}^{\prime}=M_{A B}^{\prime} \frac{l-x}{l}+M_{B A}^{\prime} \frac{x}{l} .
$$

$$
T \doteq \int_{0}^{l} M_{x} \frac{M_{A B}^{\prime}}{l}(l-x) d x+\int_{0}^{l} M_{x} \frac{M_{B A}^{\prime}}{l} x d x
$$

By use of the scale of $x$ given in Fig. 5, for both of these integrals the orthogonality foci $F_{A B}$ and $F_{B A}$ can be determined graphically. If $M_{A B}^{*}$ and $M_{B A}^{*}$ denote the ordinates of these foci, then

$$
T=M_{A B}^{*}\left(\frac{1}{2} M_{A B}^{\prime} l\right)+M_{B A}^{*}\left(\frac{1}{2} M_{B A}^{\prime} l\right)
$$

or

$$
T=\frac{1}{2} l\left(M_{A B}^{*} M_{A B}^{\prime}+M_{B A}^{*} M_{B A}^{\prime}\right) .
$$

$M_{. A B}^{*}$ and $M_{B A}^{*}$ are called the focal moments. 